\title{
ORBITAL ORIENTATIONS OF EXOPLANETS: HAT-P-4b IS PROGRADE AND HAT-P-14b IS RETROGRADE
}

\author{
Joshua N. Winn ${ }^{1}$, Andrew W. Howard ${ }^{2}$, John Asher Johnson ${ }^{3}$, Geoffrey W. Marcy ${ }^{2}$, \\ Howard IsaAcson ${ }^{2}$, Avi ShPorer ${ }^{4,5}$, Gáspár Á. BaKos ${ }^{6}$, Joel D. Hartman ${ }^{6}$, \\ Matthew J. Holman ${ }^{6}$, Simon Albrecht ${ }^{1}$, Justin R. CRepP ${ }^{3}$, And Timothy D. Morton ${ }^{3}$ \\ ${ }^{1}$ Department of Physics and Kavli Institute for Astrophysics and Space Research, Massachusetts Institute of Technology, Cambridge, MA 02139, USA \\ ${ }^{2}$ Department of Astronomy, University of California, Mail Code 3411, Berkeley, CA 94720, USA \\ ${ }^{3}$ Department of Astrophysics and NASA Exoplanet Science Institute, California Institute of Technology, MC 249-17, Pasadena, CA 91125, USA \\ ${ }^{4}$ Las Cumbres Observatory Global Telescope Network, 6740 Cortona Drive, Suite 102, Santa Barbara, CA 93117, USA \\ ${ }^{5}$ Department of Physics, Broida Hall, University of California, Santa Barbara, CA 93106, USA \\ ${ }^{6}$ Harvard-Smithsonian Center for Astrophysics, 60 Garden Street, Cambridge, MA 02138, USA \\ Received 2010 October 6; accepted 2010 December 3; published 2011 January 13
}

\begin{abstract}
We present observations of the Rossiter-McLaughlin effect for two exoplanetary systems, revealing the orientations of their orbits relative to the rotation axes of their parent stars. HAT-P-4b is prograde, with a sky-projected spin-orbit angle of $\lambda=-4.9 \pm 11.9 \mathrm{deg}$. In contrast, HAT-P-14b is retrograde, with $\lambda=189.1 \pm 5.1 \mathrm{deg}$. These results conform with a previously noted pattern among the stellar hosts of close-in giant planets: hotter stars have a wide range of obliquities and cooler stars have low obliquities. This, in turn, suggests that three-body dynamics and tidal dissipation are responsible for the short-period orbits of many exoplanets. In addition, our data revealed a third body in the HAT-P-4 system, which could be a second planet or a companion star.
\end{abstract}

Key words: planetary systems - planets and satellites: formation - planet-star interactions - stars: rotation

Online-only material: color figures, machine-readable and VO tables

\section{INTRODUCTION}

The Rossiter-McLaughlin (RM) effect, a spectroscopic phenomenon that occurs during stellar eclipses, has recently been used to study spin-orbit alignment for transiting exoplanets. Although the first nine published results suggested that the orbits of close-in planets are all well-aligned with the equatorial planes of their parent stars (Fabrycky \& Winn 2009), the next 20 results were more diverse, including orbits highly inclined with respect to the star's equatorial plane (see, e.g., Hébrard et al. 2008; Winn et al. 2009c; Johnson et al. 2009) and even retrograde orbits (Anderson et al. 2010; Narita et al. 2009; Winn et al. 2009b; Triaud et al. 2010).

These results have been marshalled as evidence against the standard scenario for planet migration, in which disk-planet tidal interactions cause the planet to spiral inward. Instead the results suggest that many close-in giant planets arrived at their current locations through gravitational perturbations from other massive bodies, followed by tidal dissipation (Triaud et al. 2010; Winn et al. 2010a; Matsumura et al. 2010). Another possibility is that protoplanetary disks are frequently misaligned with the rotation of their host stars (Bate et al. 2010; Lai et al. 2010).

Recently, a possible trend emerged from the results: misaligned systems tend to have stars with effective temperatures exceeding about $6250 \mathrm{~K}$, or masses $\gtrsim 1.2 M_{\odot}$. The evidence for this pattern is based not only on RM observations (Winn et al. 2010a) but also on the line-of-sight stellar rotation velocities of transit hosts (Schlaufman 2010). This trend may indicate that planet formation and migration are fundamentally different for low-mass stars than for high-mass stars, for which there is already evidence in the distributions of planet mass and period (Bowler et al. 2010). Another possibility is that the formation and migration processes are similar, but that the subsequent tidal evolution is different (Winn et al. 2010a). In this hypothesis, cool stars are observed to have low obliquities because tidal evolution drove them into alignment, while hot stars retain their "primordial" obliquities because of their thinner (or absent) outer convection zones and consequently slower rates of tidal dissipation.

Although the trend seems clear, it is difficult to assess its true significance because many possible variables were examined before alighting on stellar temperature and mass. The only way to be sure is to gather more data. This paper presents results for the next two systems we observed after the trend had been identified. Both systems have short-period giant planets, but HAT-P-4 is "cool" ( $T_{\text {eff }}=5860 \pm 80 \mathrm{~K}$; Kovács et al. 2007) while HAT-P-14 is "hot" $(6600 \pm 90 \mathrm{~K}$; Torres et al. 2010). We present the observations of these systems in Section 2, the analysis and results in Section 3, and a discussion in Section 4.

\section{OBSERVATIONS}

Our spectroscopic observations employed the High Resolution Spectrograph (HIRES; Vogt et al. 1994) of the Keck I 10 m telescope, on Mauna Kea, Hawaii. We gathered 35 spectra of HAT-P-4 on the night of 2010 March 29/30, and 44 spectra of HAT-P-14 on the night of 2010 April 27/28, in both cases spanning a predicted transit of the planet. An additional 14 spectra of HAT-P-4 were gathered on other nights, at essentially random orbital phases.

We used the standard instrument settings and observing procedures of the California Planet Search (Howard et al. 2009). The iodine gas absorption cell was used to track the instrumental response and wavelength scale. The relative radial velocity (RV) of each spectrum was measured with respect to an iodine-free template spectrum, using a descendant of the algorithm of Butler et al. (1996). Measurement errors were estimated from the scatter among the fits to individual spectral segments spanning a few Angstroms. Tables 1 and 2 give all the Keck/HIRES RVs, including re-reductions of those presented earlier by Kovács et al. (2007) and Torres et al. (2010). 
Table 1

Relative Radial Velocity Measurements of HAT-P-14

\begin{tabular}{ccc}
\hline \hline HJD & RV $\left(\mathrm{m} \mathrm{s}^{-1}\right)$ & Error $\left(\mathrm{m} \mathrm{s}^{-1}\right)$ \\
\hline 2454602.85804 & -187.65 & 3.64 \\
2454603.10267 & -198.11 & 3.64 \\
2454603.86302 & -142.90 & 3.70 \\
2454604.09555 & -81.34 & 4.05 \\
2454633.99342 & 212.00 & 4.03 \\
2454634.93451 & -93.25 & 3.85 \\
\hline
\end{tabular}

Notes. The RV was measured relative to an arbitrary template spectrum; only the differences are significant. The uncertainty given in Column 3 is the internal error only and does not account for any possible "stellar jitter."

(This table is available in its entirety in machine-readable and Virtual Observatory (VO) forms in the online journal. A portion is shown here for guidance regarding its form and content.)

We also conducted photometric observations of HAT-P-4, in order to refine the transit ephemeris and other system parameters. We observed the transit of 2010 March 29/30 (the same night as the Keck observations) with the Faulkes Telescope North (FTN) 2 m telescope, on Mauna Haleakala, Hawaii. We used the Spectral Instruments camera with an SDSS $i$ filter and $2 \times 2$ binning, giving a pixel scale of 0.304 and a 10.5 field of view. Unfortunately, the guider malfunctioned that night. The transit of 2010 May 7/8 was observed with the Fred L. Whipple Observatory (FLWO) $1.2 \mathrm{~m}$ telescope on Mt. Hopkins, Arizona. We used KeplerCam with an SDSS $i$ filter and $2 \times 2$ binning, giving a pixel scale of $0{ }^{\prime} 67$ and a 23!1 field of view. After standard debiasing and flat-fielding operations, we performed differential aperture photometry of HAT-P-4 and several other stars in the field.

\section{ANALYSIS \\ 3.1. HAT-P-14}

We begin with HAT-P-14, for which the analysis proved simpler. The model for the RV data took the form

$$
V_{\text {calc }}(t)=V_{\text {orb }}(t)+V_{\mathrm{RM}}(t)+\gamma,
$$

where $V_{\text {orb }}(t)$ is the radial component of a Keplerian orbit, $V_{\mathrm{RM}}(t)$ is the anomalous velocity due to the RM effect, and $\gamma$ is an arbitrary offset related to the barycentric RV of the template spectrum. To model the RM effect, we used the technique of Winn et al. (2005), which entails the construction and Doppler analysis of simulated spectra exhibiting the RM effect. The resulting formula for the anomalous velocity was

$$
V_{\mathrm{RM}}(t)=\Delta f(t) v_{p}(t)\left[1.58-0.883\left(\frac{v_{p}(t)}{8.4 \mathrm{~km} \mathrm{~s}^{-1}}\right)^{2}\right],
$$

where $\Delta f$ is the fractional loss of light during the transit, $v_{p}$ is the subplanet velocity (defined as the line-of-sight component of the stellar rotation velocity at the position of the photosphere directly behind the center of the planet), and the figure of $8.4 \mathrm{~km} \mathrm{~s}^{-1}$ is the value of $v \sin i_{\star}$ estimated by Torres et al. (2010). In calculating $\Delta f$, we adopted a linear limb-darkening law with a coefficient of 0.6288 , based on interpolation of the tables of Claret (2004). In calculating $v_{p}(t)$, we neglected differential rotation, and allowed the stellar rotation axis and the orbit normal to be separated by an angle $\lambda$ on the sky plane. For
Table 2

Relative Radial Velocity Measurements of HAT-P-4

\begin{tabular}{ccc}
\hline \hline HJD UTC & RV $\left(\mathrm{m} \mathrm{s}^{-1}\right)$ & Error $\left(\mathrm{m} \mathrm{s}^{-1}\right)$ \\
\hline 2454186.98523 & 55.67 & 2.44 \\
2454187.11242 & 52.15 & 2.12 \\
2454188.01161 & -68.10 & 2.15 \\
2454188.07151 & -75.14 & 1.92 \\
2454189.00175 & -62.98 & 2.36 \\
2454189.08264 & -46.17 & 2.18 \\
\hline
\end{tabular}

Notes. The RV was measured relative to an arbitrary template spectrum; only the differences are significant. The uncertainty given in Column 3 is the internal error only and does not account for any possible "stellar jitter."

(This table is available in its entirety in machine-readable and Virtual Observatory (VO) forms in the online journal. A portion is shown here for guidance regarding its form and content.)

a diagram of the coordinate system, see Ohta et al. (2005) or Fabrycky \& Winn (2009).

Many of the parameters of the Keplerian orbit and of the eclipses have been tightly constrained by previous observations. We adopted priors on those parameters, to keep the number of free parameters in our model to a minimum. Specifically, we used a fitting statistic

$$
\begin{aligned}
\chi^{2}= & \sum_{i=1}^{44}\left[\frac{V_{\text {obs }}\left(t_{i}\right)-V_{\text {calc }}\left(t_{i}\right)}{\sigma_{V}}\right]^{2} \\
& +\left(\frac{T_{c}-2454875.28938}{0.00047}\right)^{2}+\left(\frac{P_{\text {days }}-4.627669}{0.000005}\right)^{2} \\
& +\left(\frac{T_{\text {days }}-0.0912}{0.0017}\right)^{2}+\left(\frac{\tau_{\text {days }}-0.0287}{0.0026}\right)^{2} \\
& +\left(\frac{R_{p} / R_{\star}-0.0805}{0.0015}\right)^{2}+\left(\frac{K_{\star}-219.0 \mathrm{~m} \mathrm{~s}^{-1}}{3.3 \mathrm{~m} \mathrm{~s}^{-1}}\right)^{2} \\
& +\left(\frac{v \sin i_{\star}-8.4 \mathrm{~km} \mathrm{~s}^{-1}}{0.50 \mathrm{~km} \mathrm{~s}^{-1}}\right)^{2}
\end{aligned}
$$

where the first term is the usual sum of squared residuals and the other terms enforce Gaussian priors. In this expression, $P_{\text {days }}$ is the orbital period in days, $T_{c}$ is a particular time of inferior conjunction (in the $\mathrm{HJD}_{\mathrm{UTC}}$ system), $T_{\text {days }}$ is the time between first and fourth contact, $\tau_{\text {days }}$ is the time between first and second contact, $R_{p} / R_{\star}$ is the planet-to-star radius ratio, $K_{\star}$ is the $\mathrm{RV}$ semiamplitude of the star's Keplerian orbit, and $v \sin i_{\star}$ is the line-of-sight component of the star's equatorial rotation velocity. All the numerical values and uncertainties are taken from Torres et al. (2010). We held constant the orbital eccentricity $e=0.107$ and argument of pericenter $\omega=94^{\circ}$, since those parameters have little effect on the model once the transit ephemeris is specified.

For $\sigma_{V}$, we used the quadrature sum of the internally estimated measurement error (typically $4 \mathrm{~m} \mathrm{~s}^{-1}$; see Table 1) and a "jitter" term of $7.8 \mathrm{~m} \mathrm{~s}^{-1}$, a value determined from the condition $\chi^{2}=$ $N_{\text {dof }}$. Thus, the excess noise was assumed to be uncorrelated in time. It is comparable in magnitude to the jitter term of $7.3 \mathrm{~m} \mathrm{~s}^{-1}$ used by Torres et al. (2010).

There were only two completely free parameters in our model: $\gamma$, the overall RV offset, and $\lambda$, the projected spinorbit angle. Parameter optimization and error estimation were achieved with a Markov Chain Monte Carlo (MCMC) 

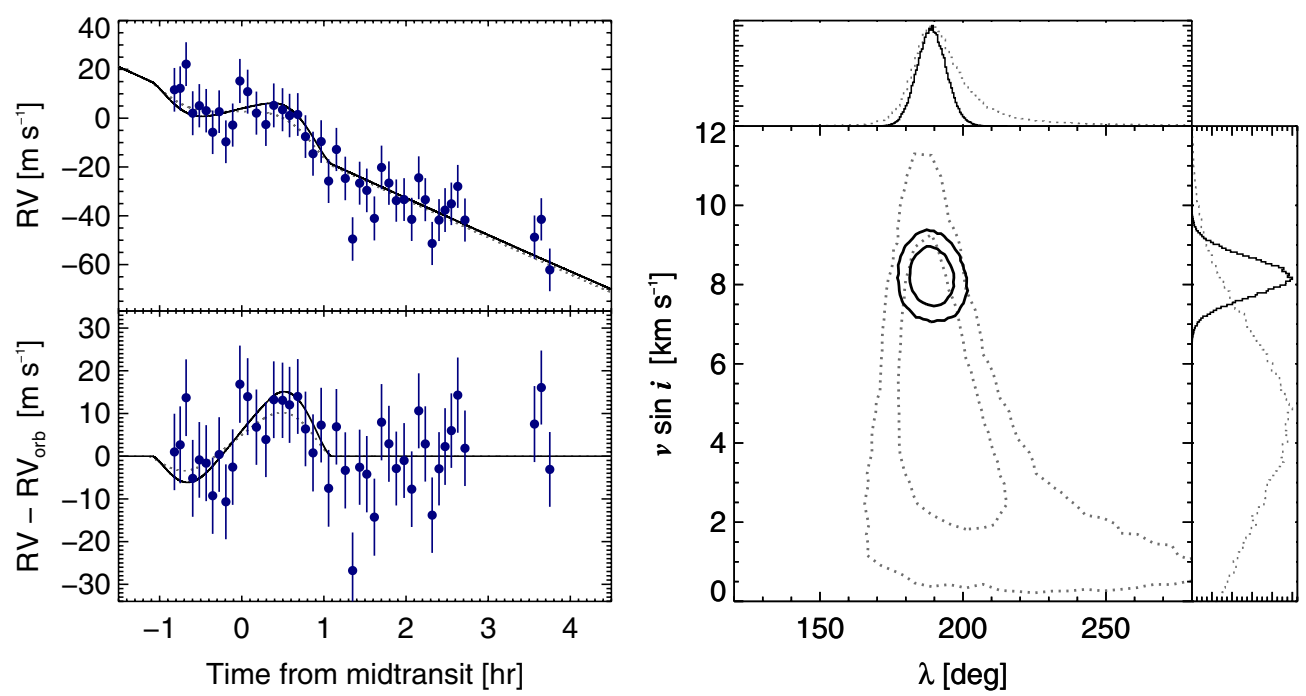

Figure 1. Results for HAT-P-14. Left panel: apparent RV variation on the night of 2010 April 27/28, spanning a transit. The top panel shows the observed RVs. For the bottom panel, the best-fitting orbital model was subtracted, thereby isolating the anomalous RV due to the RM effect. The black curve shows the best-fitting model with a prior constraint $v \sin i_{\star}=8.4 \pm 0.5 \mathrm{~km} \mathrm{~s}^{-1}$, and the dotted curve is the best-fitting model with no prior constraint on $v \sin i_{\star}$. Right panel: joint constraints on $\lambda$ and $v \sin i_{\star}$. The contours represent $68.3 \%$ and $95.4 \%$ confidence limits. The marginalized posterior probability distributions are shown on the sides of the contour plot. The solid and dotted curves show the results with and without the prior constraint on $v \sin i_{\star}$.

(A color version of this figure is available in the online journal.)

Table 3

Parameters for HAT-P-14

\begin{tabular}{lc}
\hline \hline \multicolumn{1}{c}{ Parameter } & Value \\
\hline Model Parameters & \\
\hline Projected spin-orbit angle, $\lambda[\mathrm{deg}]$ & $189.1 \pm 5.1$ \\
Projected stellar rotation rate, $v \sin i_{\star}\left[\mathrm{km} \mathrm{s}^{-1}\right]$ & $8.18 \pm 0.49$ \\
RV offset [m s $\left.{ }^{-1}\right]$ & $20.6 \pm 1.9$ \\
Velocity semiamplitude, $K_{\star}\left[\mathrm{m} \mathrm{s}^{-1}\right]$ & $218.9 \pm 5.7$ \\
Orbital period, $P$ [days] & $4.6276690 \pm 0.0000050$ \\
Mid-transit time [HJDUTC] & $0.0800 \pm 0.0015$ \\
Planet-to-star radius ratio, $R_{p} / R_{\star}$ & $83.52 \pm 0.22$ \\
Orbital inclination, $i$ [deg] & $0.1127 \pm 0.0033$ \\
Fractional stellar radius, $R_{\star} / a$ & \\
\hline Other Parameters (derived from model parameters, or from elsewhere) & \\
\hline Transit duration, first to fourth contact [days] & $0.0910 \pm 0.0035$ \\
Transit ingress or egress duration [days] & $0.0294 \pm 0.0029$ \\
Transit impact parameter & $0.894 \pm 0.013$ \\
Orbital eccentricity (Torres et al. 2010) & $0.107 \pm 0.013$ \\
Argument of pericenter [deg] (Torres et al. 2010) & $94 \pm 4$ \\
Stellar mass, $M_{\star}\left[M_{\odot}\right.$ (Torres et al. 2010) & $1.386 \pm 0.045$ \\
Stellar radius, $R_{\star}\left[R_{\odot}\right]$ & $1.468 \pm 0.042$ \\
Planetary mass, $M_{p}\left[M_{\text {Jup }}\right]$ & $2.232 \pm 0.058$ \\
Planetary radius, $R_{p}\left[R_{\text {Jup }}\right]$ & $1.142 \pm 0.033$ \\
\hline
\end{tabular}

algorithm, using Gibbs sampling and Metropolis-Hastings stepping. Table 3 summarizes the results. The first section lists all of the adjustable model parameters, for which uniform priors were adopted. The last section gives some results for other quantities that were computed based on the model parameters, or taken from Torres et al. (2010). The quoted values and ranges are based on the $50 \%, 15.85 \%$, and $84.15 \%$ confidence levels of the marginalized posteriors. Figure 1 shows the RV data and the results for $v \sin i_{\star}$ and $\lambda$.

The result for $\lambda$ is $189.1 \pm 5.1 \mathrm{deg}$, indicating that the directions of orbital motion and stellar rotation are nearly opposite as projected on the sky. This result could be anticipated from a visual inspection of Figure 1, which shows that the anomalous RV was a redshift in the second half of the transit and (less obviously) a blueshift in the first half. This is an inversion of the more familiar pattern of a well-aligned system. The orbit of HAT-P-14b is strongly misaligned with the rotational plane of its parent star.

Although the finding of a retrograde orbit is robust, the small uncertainty in $\lambda$ depends critically on the prior constraint on $v \sin i_{\star}$. If that constraint is dropped, a slower rotation rate is favored $\left(v \sin i_{\star}=4.5 \pm 2.4 \mathrm{~km} \mathrm{~s}^{-1}\right)$, allowing a broader range of spin-orbit angles $\left(\lambda=192.0_{-8.7}^{+15.6} \mathrm{deg}\right)$. These results are also illustrated by the dotted lines in Figure 1. One possible reason for the smaller result for $v \sin i_{\star}$ is differential rotation: the RM effect depends on the rotation rate over the range of latitudes spanned by the transit chord, which may differ from the spectroscopically estimated equatorial rotation rate. The 
near-grazing transit of HAT-P-14, in particular, may produce the most extreme possible difference. Further observations with a higher signal-to-noise ratio $(\mathrm{S} / \mathrm{N})$ might be able to identify the specific signal of differential rotation (Gaudi \& Winn 2007).

\subsection{HAT-P-4}

The case of HAT-P-4 was more complicated, partly because our RV observations revealed a third body in the system. Figure 2 illustrates that a single planet on a circular orbit no longer provides a satisfactory description of the data. The bestfitting model has $\chi^{2}=925.7$ with 18 degrees of freedom. The residuals have an rms of $15.9 \mathrm{~m} \mathrm{~s}^{-1}$ and are highly correlated, with almost all of the most recent RVs lying above the model curve. Allowing the orbit to be eccentric reduces $\chi^{2}$ to 906.0, but the residuals still have an rms of $15 \mathrm{~m} \mathrm{~s}^{-1}$ and show the same pattern.

A much better fit is obtained when the circular orbit is supplemented by a constant acceleration $\dot{\gamma}$. This model, illustrated in the lower panel of Figure 2, gives $\chi^{2}=137.0$ and more randomly scattered residuals with an rms residual of $6.8 \mathrm{~m} \mathrm{~s}^{-1}$. An MCMC analysis gives $\dot{\gamma}=0.0246 \pm 0.0026 \mathrm{~m} \mathrm{~s}^{-1}$ day $^{-1}$.

The constant acceleration may represent the RV variation due to a companion star or planet whose orbit is longer than the three-year span of our observations. Assuming it is a low-mass body on a nearly circular orbit, we may set $\dot{\gamma} \sim G M_{c} \sin i_{c} / a_{c}^{2}$, giving an order-of-magnitude constraint

$$
\left(\frac{M_{c} \sin i_{c}}{M_{\mathrm{Jup}}}\right)\left(\frac{a_{c}}{10 \mathrm{AU}}\right)^{-2} \sim 5.03 \pm 0.53
$$

However, given the limited time sampling of our data it is also possible that the companion has a shorter period. We have not found any compelling two-planet models, but we are continuing to gather additional RV data and will report elsewhere on the results. The pertinent conclusions for this study are (1) there is a third body in the system and (2) the mass and orbital parameters of HAT-P-4b are subject to systematic errors due to the unknown influence of the third body on the RV data. For the latter reason, in our analysis of the transit data we did not employ a prior constraint on $K_{\star}$, as we did for HAT-P-14.

Another difference in our analysis is that we fitted the available photometric data along with the transit-night RV data, because the new data offer better constraints on the transit ephemeris, depth, duration, and partial duration. Our photometric model was taken from the Transit Light Curve project (see, e.g., Holman et al. 2006; Winn et al. 2009a). In brief, we used the Mandel \& Agol (2002) formulae for a quadratic limb-darkening law, as implemented by Pál (2008). The linear coefficient was allowed to vary freely, and the quadratic coefficient was held fixed at the Claret (2004) value $(0.3418$ for $i$ band, and 0.3395 for $z$ band). The out-of-transit magnitude was allowed to be a linear function of airmass, to account for color-dependent differential extinction. The errors for each light curve were set equal to $\beta \sigma_{1}$, where $\sigma_{1}$ is the root-mean-squared (rms) residual and $\beta$ accounts for time correlations, using the method of Pont et al. (2006) as implemented by Winn et al. (2009a). Averaging times of 10-30 minutes were used to compute $\beta$, giving results (in chronological order) of 1.01, 1.73, 1.69, and 1.01. Parameter estimation was performed by the MCMC method. The photometric data and the best-fitting models are plotted in Figure 3.


Figure 2. Evidence for a third body in the HAT-P-4 system. Top panel: relative RV as a function of time. Squares represent data from 2007, and circles represent data from 2010. Below the data are the residuals between the data and the bestfitting model involving a single planet on a circular orbit. Middle panel: same, but plotted as a function of the orbital phase of the planet in days. The residuals from 2010 are systematically higher than those from 2007, which is evidence for an excess radial acceleration and hence an additional gravitating body in the HAT-P-4 system. Bottom panel: same as the middle panel, but for a model which also includes a free parameter $\dot{\gamma}$ representing a constant acceleration. The result was $\dot{\gamma}=0.0246 \pm 0.0026 \mathrm{~m} \mathrm{~s}^{-1}$ day $^{-1}$.

(A color version of this figure is available in the online journal.) 


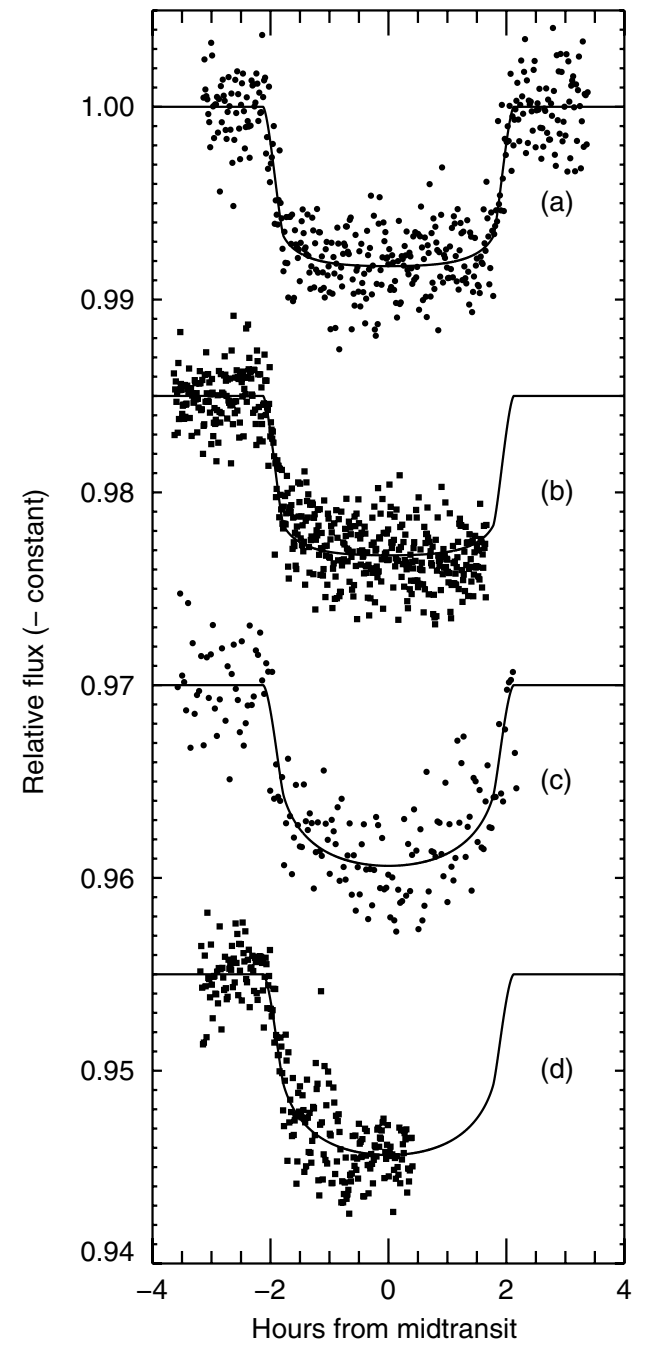

Figure 3. Transit light curves of HAT-P-4. Panels (a) and (b): FLWO $z$-band data from Kovács et al. (2007). Panel (c): FTN $i$-band data from our new observations. These data were obtained simultaneously with the Keck RM observations. Panel (d): FLWO $i$-band data from our new observations. The best-fitting model light curves are overplotted.

The formula for the anomalous velocity was

$$
V_{\mathrm{RM}}(t)=\Delta f(t) v_{p}(t)\left[1.36-0.628\left(\frac{v_{p}(t)}{5.5 \mathrm{~km} \mathrm{~s}^{-1}}\right)^{2}\right],
$$

and the fitting statistic was

$$
\begin{aligned}
\chi^{2}= & \sum_{i=1}^{35}\left[\frac{V_{\mathrm{obs}}\left(t_{i}\right)-V_{\mathrm{calc}}\left(t_{i}\right)}{\sigma_{V}}\right]^{2}+\sum_{i=1}^{1798}\left[\frac{f_{\mathrm{obs}}\left(t_{i}\right)-f_{\mathrm{calc}}\left(t_{i}\right)}{\sigma_{f}}\right]^{2} \\
& +\left(\frac{v \sin i_{\star}-5.50 \mathrm{~km} \mathrm{~s}^{-1}}{0.55 \mathrm{~km} \mathrm{~s}^{-1}}\right)^{2}
\end{aligned}
$$

using terminology similar to that of Equation (3). The only free parameters that were wholly dependent on the RV data were $\gamma$, $\lambda$, and $K_{\star}$. The jitter term was $\sigma_{V}=5.2 \mathrm{~m} \mathrm{~s}^{-1}$.

Table 4 gives the results, based on the $15.85 \%, 50 \%$, and $84.15 \%$ confidence levels of the marginalized a posteriori distributions. Figure 4 shows the RV data, and the posteriors for the RM parameters $v \sin i_{\star}$ and $\lambda$. In particular, the projected spin-orbit angle is $\lambda=-4.9 \pm 11.9 \mathrm{deg}$, consistent with good alignment between the rotational and orbital angular momentum.

As before, the quantitative results hinge on the prior constraint on $v \sin i_{\star}$. By repeating the analysis with no such constraint, we find $v \sin i_{\star}=6.4_{-0.7}^{+4.7} \mathrm{~km} \mathrm{~s}^{-1}$ and $\lambda=-11_{-39}^{+29} \mathrm{deg}$. These much broader results are also illustrated by the gray lines in Figure 4 . The only well-constrained combination of those two parameters is $v \sin i_{\star} \cos \lambda=5.77 \pm 0.41 \mathrm{~km} \mathrm{~s}^{-1}$.

\section{DISCUSSION}

It might seem surprising that tighter bounds on $\lambda$ were obtained for HAT-P-14 than for HAT-P-4, given that the $\mathrm{S} / \mathrm{N}$ of the RM effect is higher for HAT-P-4. This is a consequence of the difference in the impact parameter (the minimum sky-projected distance between the planet and the star, in units of the stellar radius). The interpretation of the RM signal is most robust for systems with a high impact parameter, because in such cases the two key parameters $v \sin i_{\star}$ and $\lambda$ play distinct roles: $v \sin i_{\star}$ controls the amplitude of the signal and $\lambda$ controls its shape (i.e., the phase of the transit when the anomalous RV switches from positive to negative, or vice versa). For systems with low impact parameters, such as HAT-P-4, the shape of the RM signal is nearly independent of $\lambda$ and both parameters control the amplitude. This leads to a strong degeneracy between those two parameters (Gaudi \& Winn 2007).

Therefore, an external constraint on $v \sin i_{\star}$ is crucial for the determination of $\lambda$ in systems with low impact parameters. In our study, we have used a prior based on the line broadening observed in the star's optical spectrum. However, it must be acknowledged that the resulting estimate of $v \sin i_{\star}$ is subject to systematic error due to uncertainties in the competing effects of macroturbulence and other broadening mechanisms. This is especially problematic for cool, low-mass stars for which turbulent and instrumental broadening exceed rotational broadening; examples of such systems are TrES-1 (Narita et al. 2007) and WASP-4 (Triaud et al. 2010). For HAT-P-4 the situation is better because rotational broadening is expected to be at least as important as turbulent broadening. ${ }^{7}$

We turn now to the question posed at the beginning of this paper: do hot stars have high obliquities? Specifically, among the host stars of close-in planets, are those with $T_{\text {eff }} \lesssim 6250 \mathrm{~K}$ more likely to be aligned with the planetary orbits than hotter stars? We have found that HAT-P-4 is a well-aligned cool star $\left(\lambda=-4.9 \pm 11.9 \mathrm{deg}, T_{\text {eff }}=5860 \pm 80 \mathrm{~K}\right)$, and HAT-P-14 is a misaligned hot star $\left(\lambda=189.1 \pm 5.1 \mathrm{deg}, T_{\text {eff }}=6600 \pm 90 \mathrm{~K}\right)$. Therefore, these new data strengthen the trend that was observed by Winn et al. (2010a).

Schlaufman (2010) found a similar pattern using a different technique, involving a comparison between the observed and expected line-of-sight rotational velocities of the stars with transiting planets. He described the pattern in terms of stellar mass rather than effective temperature. Indeed, those two parameters are strongly correlated for dwarf stars, with scatter due to metallicity and age. For HAT-P-4 and HAT-P-14, the stellar masses are $1.26_{-0.14}^{+0.06} M_{\odot}$ and $1.386 \pm 0.045 M_{\odot}$, respectively. For these systems there is a clearer contrast in effective temperature $(6.1 \sigma)$ than mass $(1.7 \sigma)$. The very different results for $\lambda$ suggest that effective temperature is more closely related to obliquity than

\footnotetext{
7 Given HAT-P-4's approximate spectral type of F7/G0, the expected macroturbulent velocity is $\approx 4.5 \mathrm{~m} \mathrm{~s}^{-1}$ (Gray 2008, p. 443) as compared to the inferred $v \sin i_{\star}$ of $5.5 \pm 0.5 \mathrm{~m} \mathrm{~s}^{-1}$.
} 



Figure 4. Results for HAT-P-4. Left panel: observed RV variation on the night of 2010 March 29/30, spanning a transit. The top panel shows the observed RVs. For the bottom panel, the best-fitting orbital model was subtracted, thereby isolating the anomalous RV due to the RM effect. The solid black curve shows the best-fitting model with a prior constraint $v \sin i_{\star}=5.5 \pm 0.5 \mathrm{~km} \mathrm{~s}^{-1}$. The best-fitting model with no prior constraint on $v \sin i_{\star}$ is plotted with a dotted curve, although it is hard to distinguish from the solid curve. Right panel: joint constraints on $\lambda$ and $v \sin i_{\star}$. The contours represent $68.3 \%$ and $95.4 \%$ confidence limits. The marginalized posterior probability distributions are shown on the sides of the contour plot. The solid and dotted curves show the results with and without the prior constraint on $v \sin i_{\star}$.

(A color version of this figure is available in the online journal.)

Table 4

Parameters for HAT-P-4

\begin{tabular}{lc}
\hline \hline \multicolumn{1}{c}{ Parameter } & Value \\
\hline Model Parameters & \\
\hline Projected spin-orbit angle, $\lambda[\mathrm{deg}]$ & $-4.9 \pm 11.9$ \\
Projected stellar rotation rate, $v \sin i_{\star}\left[\mathrm{km} \mathrm{s}^{-1}\right]$ & $5.83 \pm 0.35$ \\
RV offset $\left[\mathrm{m} \mathrm{s}^{-1}\right.$ ] & $-5.7 \pm 1.3$ \\
Velocity semiamplitude, $K_{\star}\left[\mathrm{m} \mathrm{s}^{-1}\right]$ & $66.9 \pm 8.1$ \\
Orbital period, $P$ [days] & $3.0565195 \pm 0.0000025$ \\
Mid-transit time [HJDUTC $]$ & $285.03216 \pm 0.00073$ \\
Planet-to-star radius ratio, $R_{p} / R_{\star}$ & $0.08697_{-0.00045}^{+0.00052}$ \\
Orbital inclination, $i[$ deg] & $88.76_{-1.38}^{+0.89}$ \\
Fractional stellar radius, $R_{\star} / a$ & $0.1690_{-0.00651}^{+0.0064}$ \\
\hline Other Parameters (derived from model parameters, or from elsewhere) & \\
\hline Transit duration, first to fourth contact [days] & $0.1775_{-0.0048}^{+0.0053}$ \\
Transit ingress or egress duration [days] & $0.01465_{-0.000054}^{+0.0009}$ \\
Transit impact parameter & $0.1284_{-0.092}^{+0.137}$ \\
Orbital eccentricity & 0 (assumed) \\
Stellar mass, $M_{\star}\left[M_{\odot}\right]$ (Kovács et al. 2007) & $1.26 \pm 0.10$ \\
Stellar radius, $R_{\star}\left[R_{\odot}\right]$ & $1.617_{-0.050}^{+0.057}$ \\
Planetary mass, $M_{p}\left[M_{\text {Jup }}\right]$ & $0.556 \pm 0.068$ \\
Planetary radius, $R_{p}\left[R_{\text {Jup }}\right]$ & $1.367_{-0.044}^{+0.052}$ \\
\hline
\end{tabular}

mass. This in turn would support the hypothesis of Winn et al. (2010a) that the differing obliquities are a consequence of differing internal structure of the stars, and specifically the depth of the outer convective zone, since this structural difference is more closely related to effective temperature than mass (Pinsonneault et al. 2001).

It will be interesting to examine all the systems for which the RM effect has been measured, to see whether temperature or mass is more important, and whether there are other variables related to obliquity. We defer such a study for the future, to allow the sample size to grow substantially since the last such analysis by Winn et al. (2010a). One variable that will be especially interesting to assess is the presence or absence of a third body. A migration mechanism involving the Kozai effect, which has been invoked to explain high obliquities, requires the existence of a third body (see, e.g., Fabrycky \& Tremaine 2007). In planet-planet scattering scenarios, a third body may also be present, although it could have been ejected (see, e.g., Chatterjee et al. 2008). Evidence for additional bodies has been found in some well-aligned systems such as HAT-P-13 (Bakos et al. 2010; Winn et al. 2010b) and HAT-P-4 (this study), along with some misaligned systems such as HAT-P-7 (Winn et al. 2009c), HD 80606 (Naef et al. 2001), HAT-P-11 (Bakos et al. 2010, Winn et al. 2010c), and WASP-8 (Queloz et al. 2010). A systematic multiplicity study would be illuminating (see, e.g., Narita et al. 2010), as would a comparison between the observed obliquity distribution and that predicted by the Kozai model (Fabrycky \& Winn 2009; Morton \& Johnson 2010). 
It will also be interesting to extend these studies to multitransiting systems such as Kepler-9 (Holman et al. 2010). This will allow the mutual inclinations of the orbits to be determined, along with the obliquity of the star (Fabrycky 2009; Ragozzine \& Holman 2010). If the mechanism that causes spin-orbit misalignments is related to star formation (Bate et al. 2010) or star-disk interactions (Lai et al. 2010), and is not related to the planet, then one would expect the planetary orbits to be well aligned and the star to be tipped away from their common orbital plane. In contrast, if the close-in planet "pile-up" has an origin in dynamical scattering and tidal dissipation, then the planetary orbits would be highly inclined.

We thank Teruyuki Hirano for interesting discussions related to this work, Amaury Triaud and the anonymous referee for insightful comments on the manuscript, and John Southworth for making available his useful code JKTLD for calculating theoretical limb-darkening coefficients. We gratefully acknowledge support from the NASA Origins program through awards NNX09AD36G and NNX09AB33G, and the MIT Class of 1942. S.A. acknowledges support from an NWO Rubicon fellowship.

This paper uses observations obtained with facilities of the Las Cumbres Observatory Global Telescope. Some data presented herein were obtained at the W.M. Keck Observatory, which is operated as a scientific partnership among the California Institute of Technology, the University of California, and the National Aeronautics and Space Administration, and was made possible by the generous financial support of the W.M. Keck Foundation. We extend special thanks to those of Hawaiian ancestry on whose sacred mountain of Mauna Kea we are privileged to be guests. Without their generous hospitality, the Keck observations presented herein would not have been possible.

Facilities: Keck:I (HIRES), FLWO:1.2m (KeplerCam), FTN (Spectral)

\section{REFERENCES}

Anderson, D. R., et al. 2010, ApJ, 709, 159

Bakos, G. Á., et al. 2010, ApJ, 710, 1724
Bate, M. R., Lodato, G., \& Pringle, J. E. 2010, MNRAS, 401, 1505

Bowler, B. P., et al. 2010, ApJ, 709, 396

Butler, R. P., Marcy, G. W., Williams, E., McCarthy, C., Dosanjh, P., \& Vogt, S. S. 1996, PASP, 108, 500

Chatterjee, S., Ford, E. B., Matsumura, S., \& Rasio, F. A. 2008, ApJ, 686, 580

Claret, A. 2004, A\&A, 428, 1001

Fabrycky, D. C. 2009, in Proc. IAU Symp. 253, Transiting Planets, ed. F. Pont, D. Sasselov, \& M. Holman (Cambridge: Cambridge Univ. Press), 173

Fabrycky, D., \& Tremaine, S. 2007, ApJ, 669, 1298

Fabrycky, D. C., \& Winn, J. N. 2009, ApJ, 696, 1230

Gaudi, B. S., \& Winn, J. N. 2007, ApJ, 655, 550

Gray, D. F. 2008, The Observation and Analysis of Stellar Photospheres (Cambridge: Cambridge Univ. Press)

Hébrard, G., et al. 2008, A\&A, 488, 763

Holman, M. J., et al. 2006, ApJ, 652, 1715

Holman, M. J., et al. 2010, Science, 330, 51

Howard, A. W., et al. 2009, ApJ, 696, 75

Johnson, J. A., Winn, J. N., Albrecht, S., Howard, A. W., Marcy, G. W., \& Gazak, J. Z. 2009, PASP, 121, 1104

Kovács, G., et al. 2007, ApJ, 670, L41

Lai, D., Foucart, F., \& Lin, D. N. C. 2011, MNRAS, in press

Mandel, K., \& Agol, E. 2002, ApJ, 580, L171

Matsumura, S., Peale, S. J., \& Rasio, F. A. 2010, ApJ, 725, 1995

Morton, T., \& Johnson, J. 2010, ApJ, submitted, arXiv:1010.4025

Naef, D., et al. 2001, A\&A, 375, L27

Narita, N., Sato, B., Hirano, T., \& Tamura, M. 2009, PASJ, 61, L35

Narita, N., et al. 2007, PASJ, 59, 763

Narita, N., et al. 2010, PASJ, 62, 779

Ohta, Y., Taruya, A., \& Suto, Y. 2005, ApJ, 622, 1118

Pál, A. 2008, MNRAS, 390, 281

Pinsonneault, M. H., DePoy, D. L., \& Coffee, M. 2001, ApJ, 556, L59

Pont, F., Zucker, S., \& Queloz, D. 2006, MNRAS, 373, 231

Queloz, D., et al. 2010, A\&A, 517, L1

Ragozzine, D., \& Holman, M. J. 2010, ApJ, submitted, arXiv:1006.3727

Schlaufman, K. C. 2010, ApJ, 719, 602

Torres, G., et al. 2010, ApJ, 715, 458

Triaud, A., et al. 2010, A\&A, 524, 25

Vogt, S. S., et al. 1994, Proc. SPIE, 2198, 362

Winn, J. N., Fabrycky, D., Albrecht, S., \& Johnson, J. A. 2010a, ApJ, 718, L145

Winn, J. N., Holman, M. J., Carter, J. A., Torres, G., Osip, D. J., \& Beatty, T. 2009a, AJ, 137, 3826

Winn, J. N., Johnson, J. A., Albrecht, S., Howard, A. W., Marcy, G. W., Crossfield, I. J., \& Holman, M. J. 2009b, ApJ, 703, L99

Winn, J. N., et al. 2005, ApJ, 631, 1215

Winn, J. N., et al. 2009c, ApJ, 700, 302

Winn, J. N., et al. 2010b, ApJ, 718, 575

Winn, J. N., et al. 2010c, ApJ, 723, 223L 